\title{
Extracción Laparoscópica del Diu Intraperitoneal
}

\author{
Dr . Laureano Marín Ardila* \\ Dr. Eduardo Rodríguez de Francisco**
}

Uno de los más útiles métodos de Planificación Familiar lo constituye indudablemente el DIU.

Su fácil aplicación, inclusive por personal paramédico y sus excelentes resultados (96 - $97 \%$ de seguridad) lo han popularizado extensamente, hasta el punto de que los cálculos de utilización a nivel mundial, llegan a una cifra aproximada de 60 millones de usuarias.

La tolerancia del método, especialmente en cuanto hace referencia a sus efectos secundarios, nos hacen creer que el DIU es en la actualidad, y lo seguirá siendo por mucho tiempo, un procedimiento efectivo, seguro y de inmensa aceptación general.

En Colombia y a nivel de la institución privada cuyos programas de Planificación Familiar son de los más amplios del mundo, su aplicación prácticamente se ha limitado al Lippes en sus cuatro tamaños y al DIU de cobre, generalmente conocido como $\mathrm{T}$. de Cobre.

Eventualmente las usuarias del DIU, manifiestan discretas molestias con su utilización y tienen la tendencia a culpar al dispositivo de trastornos generales que no pueden tener relación alguna con el método en sí. Es frecuente la queja por pérdida de peso, por aumento del mismo, por cefaleas, por cambios en la pigmentación de la piel, por caí-

\footnotetext{
*. Ginecólogo, Universidad Central de Madrid - España.

** Director, Clínica Piloto, Profamilia - Bogotá.
}

da del pelo o de las uñas, etc., los cuales debidamente investigados no tienen relación alguna con el uso del dispositivo.

En ocasiones, afortunadamente más raras, algunos médicos poco prácticos en el trabajo de Planificación Familiar, insinúan y a veces ordenan a la señora el retiro del dispositivo culpando al mismo de eventuales trastornos digestivos, urinarios, ginecológicos, etc., estableciendo una situación de iatrogénesis, que conduce a la larga a la ahincada solicitud de la paciente para el retiro del DIU, y su cambio por otro método que puede no ser el adecuado de acuerdo con la edad, paridad, condiciones ginecológicas, etc.

El personal médico y paramédico que trabaja en Profamilia - Colombia, solicita de sus usuarias el que tanto la aplicación como el retiro del DIU, se lleve a cabo en plena menstruación, con el objeto de que el cérvix permita la instrumentación fácil y la maniobra no ocasione dolor ni resistencia alguna a la señora. Otra razón importante es la absoluta seguridad que tiene el médico de que la señora no se encuentre embarazada y puede programarse además para el acto quirúrgico, si desea la ligadura de trompas.

Es frecuente, sin embarqo, que el retiro solicitado por la interesada y por las razones antes aludidas, o pedido por el médico, se dificulte sustancialmente y el operador se encuentre en imposibilidad de complacer a la paciente encontrando una gran resistencia para la extracción. Se supone en tal caso que 
el DIU ha perforado parcial o totalmente la pared uterina, impidiendo en esta forma la maniobra de retiro.

En otras oportunidades, los hilos que protruyen a través del cérvix han desaparecido, sea por expulsión accidental o por introducción, también accidental dentro de la cavidad uterina. El operador en esta eventualidad, después de ubicado el cuello puede sondear instrumentalmente el interior del útero y encontrarse con un DIU imposible de percibir.

Las dos circunstancias mencionadas, solamente pueden aclararse mediante la toma de radiografías simples de abdomen en posiciones A. P. y lateral, las cuales indicarán que el IDU se encuentra en cavidad uterina y su altura y desviaciones laterales o anteroposteriores, pueden ser el indicio de perforación de la pared uterina.

Un método más sofisticado pero de mayor precisión puede ser el practicar una histerografía con medio de contraste y en varias incidencias. Este estudio simple pero indudablemente costoso puede indicar muy claramente que el DIU está fuera de la cavidad uterina.

Nosotros optamos por la práctica de radiografías simples de abdomen sin ordenar el estudio histeroqráfico. El nivel económico de nuestras usuarias, limita sustancialmente estudios costosos y sofisticados que no pueden ser costeados ni por las usuarias ni por la Institución.

En Profamilia Colombia, se lleva a cabo un intenso programa de Planificación Familiar con DIU, razón por la cual se ve abocada a frecuentes retiros de los dispositivos; en la mayoría de los casos a solicitud de la interesada o de su médico particular, por razones de orden médico, como es el caso concreto de la emigración a cavidad abdominal después de perforación de la pared uterina.

El personal de consulta externa, encuentra entonces las dificultades arriba mencionadas, cuya solución es eminentemente quirúrgica.
Vale la pena mencionar entonces que Profamilia practica en promedio de 25 a 30 laparoscopias, para esterilizaciones femeninas voluntarias en su Clínica Piloto de Bogotá, con un personal altamente calificado de 5 cirujanos laparoscopistas, 2 anestesiólogos y 9 enfermeras, diariamente.

Que en el año de 1979, solamente la Clínica Piloto practicó un total de 7.000 laparoscopias sin accidentes dignos de mención y por lo tanto se encuentra en condiciones de resolver situaciones anormales. Como es precisamente el caso de los DIU perforantes y emigrados parcial o totalmente a la cavidad abdominal.

Como pequeño recuento histórico, vale la pena mencionar que uno de nuestros ginecólogos laparoscopistas, el doctor Laureano Marín Ardila, practicando una ligadura tubaria por laparoscopia encontró sobre la cara posterior del útero, la punta de un dispositivo que no había podido ser retirado por vía vaginal; con la pinza de Palmer practicó una perforación mínima en peritoneo visceral y poco a poco logró extraer el DIU, el cual finalmente fue retirado de la cavidad abdominal al exterior sin que se presentara sangrado alguno, ni diera lugar a la más míninia complicación. En esta forma fue posible evitar el tener que practicar una laparotomía exploratoria y fue entonces cuando se propuso buscar este tipo de dispositivos emigrantes para extraerlos a través de la laparoscopia.

Ante la sospecha o seguridad de que el dispositivo se encuentra en cavidad peritoneal se prepara la paciente para un acto quirúrgico menor, como es la laparoscopia, que en este momento negará o confirmará el diagnóstico, en cuyo caso se procede a la extracción.

\section{Procedimiento}

Premedicación: Diazepán de $5 \mathrm{mg}$. una hora y media antes de la intervención y posteriormente medio miligramo de Sulfato de Atropina. En la sala de 
operación se aplica venoclisis rutinaria a través de la cual se inyectan Meperidina y Diazepán, de acuerdo con el peso de la paciente; ocasionalmente se agrega otra medicación anestésica.

Con la paciente en posición de litotomía y posteriormente en Trendelemburg de unos 40 grados, se practica un. examen ginecológico para detallar los genitales internos, etc. y poder definir mejor el procedimiento. Se coloca entonces una pinza de cuello con movilizador uterino por la parte vaginal y se inicia el procedimiento abdominal introduciendo una aguja de Tuohy o de Verres, a fin de establecer el neumoperitoneo, utilizando $\mathrm{CO}_{2}$ para un máximo de dos a tres litros en cavidad peritoneal. En el mismo lugar, o sea prácticamente bordeando el ombligo por su parte inferior, se hace una incisión de un centímetro que permita la entrada del trocar del laparoscopio, con el debido cuidado de no interesar vísceras u órganos intra-abdominales. En estas condiciones se introduce a través del trocar el laparoscopio, después de haber retirado el mandril.

En las instalaciones de Profamilia se ha utilizado el lente $\mathrm{KLI}$. de $12 \mathrm{~mm}$ de diámetro en forma de bayoneta con posibilidad de utilizar pinzas para aplicación de bandas de Yoon y pinzas de Palmer para cauterio, siendo éstas las más utilizadas para el retiro del dispositivo.

Teniendo la cavidad peritoneal bajo el control del laparoscopio el operador lo dirige hacia el lugar que previamente ha indicado la ubicación del dispositivo, bien sea por radiografía o por tacto y en su defecto se procede a la exploración general de dicha cavidad buscándolo como es lógico en los sitios de ubicación más frecuentes como son la pared uterina, principalmente hacia su borde derecho e izquierdo, fondo de Douglas y debajo de vejiga.

No obstante se han encontrado varios dispositivos sueltos sobre el epiplón o involucrándolo. Ya localizado el dispositivo se procede a su extracción, generalmente con la pinza de Palmer como ya se dijo. Se diseca inicialmen- te un extremo del dispositivo haciendo un ojal cuando el peritoneo u otras estructuras lo cubren pero lo dejan entrever $y$ por este ojal se continúa la extracción del dispositivo siguiendo su forma, con el objeto de traumatizar lo menos posible los tejidos vecinos y principalmente las arterias o venas cuàndo éstas están próximas, para evitar hemorragias. Una vez lograda la liberación del dispositivo se toma éste, por uno de sus extremos con la pinza de Palmer y teniendo en cuenta que ya está suelto en la cavidad peritoneal se procede a extraerlo sacando junto con la pinza el laparoscopio a través del trocar, el cual es fijado por la instrumentadora. Posteriormente se introduce nuevamente el laparoscopio con el objeto de revisar el lecho del dispositivo y estar seguros de que no se ha causado daño a órganos importantes y de cohibir la hemorragia si la hay, aplicando cauterio.

Para facilitar todas estas maniobras, nos es de mucha utilidad el movilizador uterino a través de la vagina, lo cual nos permite colocar el útero y sus estructuras vecinas en la posición más adecuada para poder trabajar con seguridad y más amplitud visual. Una vez terminada la maniobra y seguros de que no hay complicación importante que nos conduzca a otra intervención mayor, como no nos ha sucedido hasta el momento, extraemos el laparoscopio, el gas y el trocar y procedemos a coger un punto de piel para cerrar el orificio de entrada.

Seguidamente se retiran los instrumentos vaginales.

Inmediatamente la paciente es llevada a recuperación, en donde es controlada por unas dos o tres horas hasta cuando se sienta en capacidad de regresar a su domicilio, lógicamente en compañía de una persona adulta, preferiblemente su esposo o compañero y con las instrucciones que el caso requiere. A los ocho días se practica un control sin haber encontrado hasta el momento efectos secundarios o complicaciones dignas de mención. 
En las instalaciones de la Clínica Piloto de Profamilia - Bogotá, se han praticado hasta el momento 31 retiros de dispositivo intra-abdominal por laparoscopia, con las características que los cuadros anexos demuestran. (No ha sido necesario recurrir a la laparotomía para este procedimiento).

\section{Comentarios Generales}

Es interesante mencionar que en Profamilia Colombia se han aplicado en total 464.878 DIU y se han practicado en total 93.000 laparoscopias. De estos datos corresponden a la Clínica Piloto de Bogotá 132.150 DIU y 25.590 laparoscopias, las cuales en su gran mayoría se han realizado para esterilizaciones voluntarias.

Como dato por lo demás curioso e interesante, les quiero relatar el caso de una mujer de nivel económico muy bajo que concurre a la Clínica Piloto en solicitud de esterilización voluntaria, en vista de que le han fallado 3 dispositivos Lippes aplicados en diferentes Centros de Planificación Familiar de Bogotá.
Inicialmente pensamos que dichos dispositivos habían sido expulsados con los respectivos partos y un aborto que fue el último embarazo (G. 5, P. 4 y A.1); no obstante se le ordenó una radiografía antero posterior con la gran sorpresa de encontrar 3 dispositivos Lippes, 2 de los cuales estaban francamente en cavidad peritoneal y uno dudoso. Se le practicó laparoscopia, se le extrajeron los dos dispositivos intraperitoneales, de los cuales uno se encontraba visible a través del epiplón pero involucrándolo. Revisando el lecho, se encontró el segundo dispositivo también involucrando epiplón pero conformando un plastrón en fondo uterino, el cual se extrajo también sin complicación.

Revisando el lecho de los dos anteriores, se encontró que un tercero protruía por el fondo uterino intentándose sacar a través del laparoscopio, pero presentó dificultades, motivo por el cual se introdujo un gancho especial a través de cuello uterino, se enganchó el dispositivo y el ayudante lo retiró por vagina, bajo la observación del laparoscopio. Como es lógico, a esta paciente se le ligaron las trompas con bandas de Yoon y fue controlada a los ocho días sin presentar complicación alguna.

\begin{tabular}{|c|c|c|c|c|c|c|c|c|c|c|c|}
\hline $\begin{array}{c}\text { No. de } \\
\text { CASOS }\end{array}$ & $\begin{array}{c}\text { EDAD } \\
\text { PROMEDIO }\end{array}$ & \multicolumn{3}{|c|}{$\begin{array}{c}\text { NIVEL } \\
\text { SOCIO-ECONOMICO }\end{array}$} & \multicolumn{3}{|c|}{$\begin{array}{c}\text { PARIDAD } \\
\text { PROMEDIO }\end{array}$} & \multicolumn{4}{c|}{$\begin{array}{c}\text { MOTIVO DE } \\
\text { CONSULTA }\end{array}$} \\
\hline \multirow{2}{*}{29} & \multirow{2}{*}{26.3 Años } & A & M & B & G & P & A & Ret. D. & L. T. & Otros \\
\cline { 2 - 11 } & & 1 & 6 & 22 & 2.58 & 2.21 & 1 & 10 & 16 & 3 \\
\hline
\end{tabular}

\begin{tabular}{|l|c|c|c|c|c|c|}
\hline \multirow{2}{*}{$\begin{array}{l}\text { TIEMPO PROM. } \\
\text { UTILIZACION } \\
\text { DISPOSITIVO }\end{array}$} & \multicolumn{3}{|c|}{$\begin{array}{c}\text { HALLAZGOS DE } \\
\text { DISPOSITIVOS }\end{array}$} & \multicolumn{2}{c|}{$\begin{array}{c}\text { TIPO DE } \\
\text { DISPOSITIVOS }\end{array}$} & ANESTESIA \\
\cline { 2 - 7 } & R. X. & Lap. & Tacto & Lippes & Tcu. & $\begin{array}{c}\text { General } \\
\text { y Local }\end{array}$ \\
\hline $\begin{array}{l}2 \\
7 \text { Años y } \\
\text { meses }\end{array}$ & 6 & 21 & 3 & 29 & 2 & V. K. D. \\
\hline
\end{tabular}




\section{Resumen y Conclusiones}

1. Está demostrado que se pueden extraer los dispositivos intraperitoneales por laparoscopia.

2. La laparotomía sólo se utilizará para extraer dispositivos intraperitoneales en aquellos casos en que no se logra hacerlo con la laparoscopia.
3. La extracción del dispositivo intraperitoneal se practica en forma ambulatoria.

4. No siempre los dispositivos intrauterinos son expulsados con el parto o aborto cuando hay embarazos, hay que tener en mente la posibilidad de que pueden haber emigrado a cavidad peritoneal.

\section{LAPAROSCOPIC REMOVAL OF INTRAPERITONEAL IUD}

\section{Summary and Conclusions}

1. It has been proved that it is possible to remove intraperitoneal devices through laparoscopy.

2. Laparatomy will be used to remove intraperitoneal devices only in those cases where laparoscopy does not work.
3. Removal of intraperitoneal devices is done in an ambulatory manner.

4. Intrauterine Devices are not always thrown out during delivery or abortion in the case of pregnancies, so it is necessary to consider the possibility that they may have entered the peritoneal cavity. 